\title{
Roll-over-web Coating of Pseudoplastic and Viscoplastic Sheets Using THE LUbrication Approximation
}

\author{
Souzanna Sofou and Evan Mitsoulis* \\ School of Mining Engineering \& Metallurgy \\ National Technical University of Athens \\ Zografou, 157 80, Athens, Greece
}

\begin{abstract}
The Lubrication Approximation Theory (LAT) is used to provide numerical results in roll coating over a moving flat web. The Herschel-Bulkley model of viscoplasticity is used, which reduces with appropriate modifications to the Bingham, power-law, and Newtonian models. Results are obtained for such quantities as coating thickness, separation point, and the volumetric flow rate required for various values of the power-law index (in the case of pseudoplasticity) and of the Bingham number (in the case of viscoplasticity). All these values increase substantially with the increasing non-Newtonian character of the fluid. Yielded and unyielded areas are quantitatively shown for several cases of viscoplasticity. Pressure gradient and pressure distributions are given for all cases. Integrated quantities of engineering interest are also calculated. These include the maximum pressure, the roll/sheet separating force, and the power input to the roll. These quantities increase substantially and monotonically in a dimensionless form, as the power-law index decreases or the Bingham number increases.
\end{abstract}

KEY WORDS: roll-over-web coating, pseudoplasticity, viscoplasticity, yield stress, yield line, Herschel-Bulkley model.

\section{INTRODUCTION}

D Oll COATING IS a process used in a variety of industries - producing photographic films, paper and coated products, magnetic recording

\footnotetext{
*Author to whom correspondence should be addressed. E-mail: mitsouli@metal.ntua.gr Figures 1, 3 and 14 appear in color online: http://jpf.sagepub.com
}

Journal of PLASTIC FILM \& SHEETING, Vol. 21-Oстовer 2005 


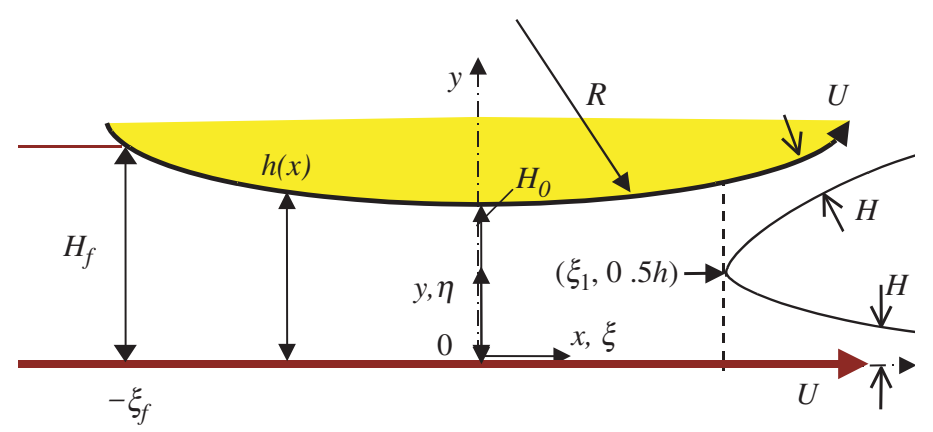

Figure 1. Schematic representation of the process of roll coating over a moving web and definition of variables.

media - for the production of thin layers of specific thickness and final appearance, coated over a thin moving sheet (sometimes referred to as the web). This procedure was theoretically introduced by Middleman [1] and concerns the case where the sheet has the same velocity as the peripheral roll speed and moves in the same direction (see Figure 1) [1].

Many materials used in roll coating are non-Newtonian, exhibiting either pseudoplastic (shear-thinning or -thickening) [1] or viscoplastic (presence of a yield stress) behavior (see, e.g., Bird et al. [2]). Models describing pseudoplastic behavior include the power-law, Carreau, Cross, etc. Models describing viscoplastic behavior include the Bingham, Herschel-Bulkley, and Casson. The Herschel-Bulkley model has the advantage of reducing - with an appropriate choice of parameters - to the Bingham, power-law, or Newtonian model. In simple shear flow, it takes the form ([2]; see also Figure 2):

$$
\begin{array}{ll}
\tau=K|\dot{\gamma}|^{n-1} \dot{\gamma} \pm \tau_{\mathrm{y}}, & \text { for }|\tau|>\tau_{\mathrm{y}}, \\
\dot{\gamma}=0, & \text { for }|\tau| \leq \tau_{\mathrm{y}},
\end{array}
$$

where $\tau$ is the shear stress, $\dot{\gamma}$ is the shear rate $(=\mathrm{d} u / \mathrm{d} y), \tau_{\mathrm{y}}$ is the yield stress, $K$ is the consistency index, and $n$ is the power-law index. Note that when $n=1$ and $K=\mu$ (a constant), the Herschel-Bulkley model reduces to the Bingham model. When $\tau_{\mathrm{y}}=0$, the power-law model is recovered, and when $\tau_{\mathrm{y}}=0$ and $n=1$, the Newtonian model is obtained.

It should be noted that in viscoplastic models, when the shear stress $\tau$ falls below $\tau_{\mathrm{y}}$, a solid structure is formed (unyielded). Also, in viscoplasticity, the yield stress can be expressed as a function of the pressure gradient and the yield line, that is, a line separating the yielded 


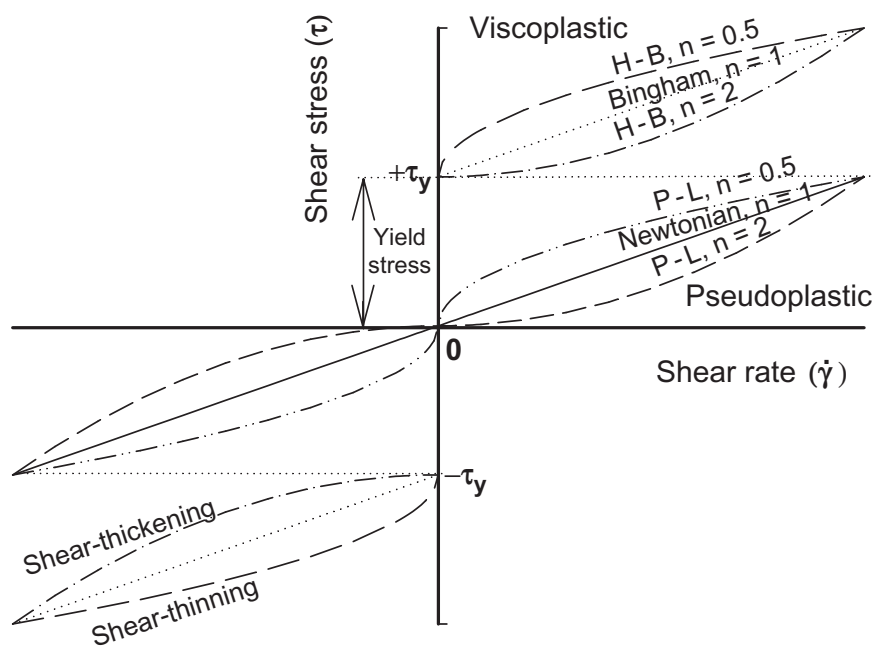

Figure 2. Shear stress vs shear rate for various pseudoplastic and viscoplastic fluids.

from the unyielded material. This equation, however, strongly depends on the geometry of the flow field.

An early analysis of roll coating has been carried out by Middleman [1] for the case of roll-over-web coating pseudoplastic fluids from an infinite reservoir. Coyle [3] presented results for both the forward [4] and reverse [5] case of roll-over-roll coating, for the asymmetric case including the effects of pseudoplasticity and viscoelasticity. It appears, therefore, that no work has been done for the process of roll-over-web coating for viscoplastic materials. It is the purpose of this work to provide numerical results for this process, for such quantities as coating thickness, separation point, and the volumetric flow rate required for both pseudoplastic and viscoplastic materials. The results will be given for full parametric studies of the power-law index and the Bingham number or dimensionless yield stress. For pseudoplastic fluids, the results of Middleman [1] are verified and extended, while for viscoplastic fluids the results are new.

\section{MATHEMATICAL MODELING}

\section{Governing Equations}

As explained by Middleman [1] and with regard to Figure 1, the lubrication approximation theory (LAT) regards locally fully developed 
shear flow between the roll and the web. The conservation of momentum then gives

$$
\frac{\mathrm{d} P}{\mathrm{~d} x}=\frac{\mathrm{d} \tau_{\mathrm{xy}}}{\mathrm{d} y}
$$

where $\tau_{\mathrm{xy}}=\tau$ is the shear stress in the transverse direction. The shear stress is given by the Herschel-Bulkley model in the present work (Equation (1)).

In roll-over-web coating, the following dimensionless parameters are introduced $[1,6]$ :

$$
\begin{aligned}
& \xi=\frac{x}{\sqrt{R H_{0}}}, \quad h=H_{0}\left(1+\frac{x^{2}}{2 R H_{0}}\right), \quad \eta=\frac{y}{H_{0}}, \quad P^{\prime}=\frac{P}{K}\left(\frac{H_{0}}{U}\right)^{n}\left(\frac{H_{0}}{R}\right)^{1 / 2}, \\
& u=\frac{u_{x}}{U}, \quad \tilde{h}=\frac{h}{H_{0}}, \quad \lambda=\frac{Q}{U H_{0}},
\end{aligned}
$$

where $\lambda$ is a dimensionless flow rate, and the rest of the symbols are defined in Figure 1.

According to Middleman [1], it is assumed that the material splits evenly at point $\left(\xi_{1}, 0.5 \mathrm{~h}\right)$ to coat both the roll and the sheet. Then the volumetric flow rate, $Q$, the coating thickness $H$, and $\lambda$ are related by

$$
Q=2 U H
$$

and

$$
\lambda=\frac{2 H}{H_{0}} .
$$

For the case of viscoplastic fluids, it is customary to define the Bingham number [7]:

$$
B n=\frac{\tau_{\mathrm{y}}}{K}\left(\frac{H_{0}}{U}\right)^{n}
$$

where $B n=0$ corresponds to purely viscous fluids and $B n \rightarrow \infty$ corresponds to purely plastic solids. 


\section{Pseudoplastic Fluids}

In the present work, we use the equations proposed by Middleman [1] for pseudoplastic power-law fluids. More precisely, the equation for the pressure gradient becomes:

$$
\frac{\mathrm{d} P^{\prime}}{\mathrm{d} \xi}=\left(\frac{2 n+1}{n}\right)^{n} 2^{n+1} \frac{\left[(\tilde{h}-\lambda)^{2}\right]^{n-1 / 2}(\tilde{h}-\lambda)}{\tilde{h}^{2 n+1}} .
$$

Moreover, the separation point, $\xi_{1}$, is found by asserting that the pressure vanishes there, at which point $u$ also vanishes [1]. Setting $u\left(\xi_{1}, 1 / 2 \tilde{h}\right)=0$, gives the separation point $\xi_{1}$ as a function of the dimensionless coating thickness, $\lambda$, and of the power-law index, $n$ :

$$
\xi_{1}=\left(2\left(\frac{2 n+1}{n} \lambda-1\right)\right)^{1 / 2} .
$$

It should be noted that the above boundary conditions used are one choice of conditions, and other types have been proposed in the literature [3-5]. The results depend on the boundary conditions assumed, but the overall trends, based on the rheology of the material, are the same.

\section{Viscoplastic Fluids}

\section{Bingham Plastics}

By integrating Equation (2), and after using Equation (3) we obtain:

$$
\pm 1+\frac{1}{B n} \frac{\mathrm{d} u}{\mathrm{~d} \eta}=-\frac{\mathrm{d} P^{\prime}}{\mathrm{d} \xi} \frac{1}{B n}\left(\eta_{0}-\eta\right),
$$

where $\eta_{0}$ is the position of zero shear stress.

From the Newtonian analysis, we know that there are three flow regions in the $x$-direction: one region near the nip, which has a negative pressure gradient $\left(\mathrm{d} P^{\prime} / \mathrm{d} \xi<0\right)$, and two other regions away from the nip at the entrance and at the exit with an opposite sign $\left(\mathrm{d} P^{\prime} / \mathrm{d} \xi>0\right)$, as shown in Figure 3(a). For viscoplastic materials, we know that there are also three flow regions in the $y$-direction: one region where the material is unyielded $\left(\eta_{p 2} \leq \eta \leq \eta_{p 1}\right)$ and two regions where it is yielded $\left(0 \leq \eta \leq \eta_{p 2}\right.$ and $\left.\eta_{p 1} \leq \eta \leq \tilde{h}\right)$, as shown in Figure 3(b). 
(a)

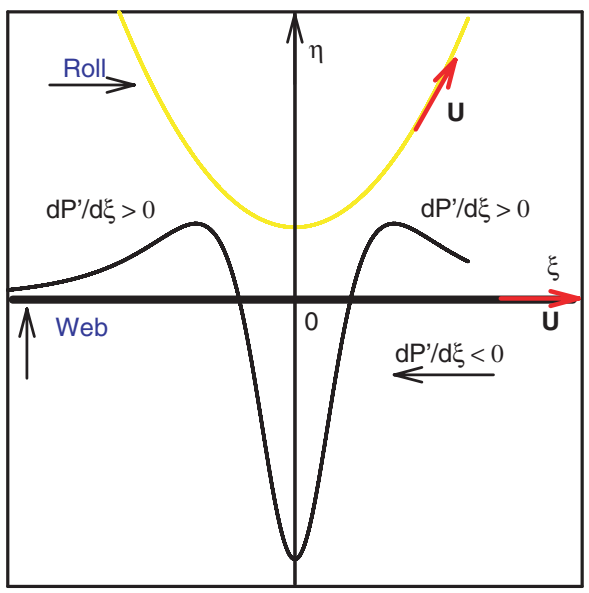

(b)

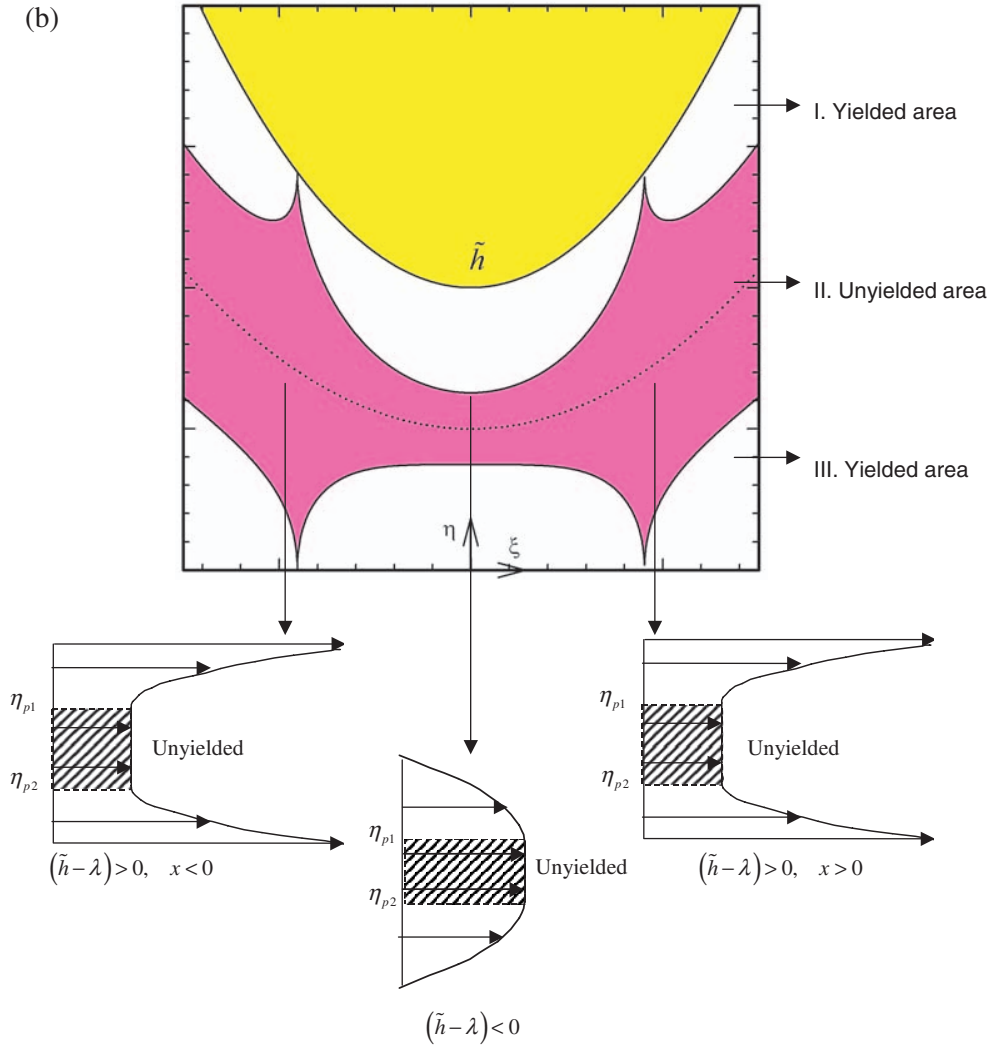

Figure 3. (a) Flow regions in the $x$-direction: pressure-gradient distribution and (b) flow regions in the $y$-direction: yielded/unyielded regions and velocity profiles. 
The location of the two yield lines, which separate the yielded from the unyielded material, can be found by setting $\mathrm{d} u / \mathrm{d} \eta=0$ in Equation (9). Thus, we obtain:

$$
\begin{array}{ll}
\eta_{p 1}=\eta_{0}+\frac{B n}{\mathrm{~d} P^{\prime} / \mathrm{d} \xi}, & \text { for } \tau=K \dot{\gamma}+\tau_{\mathrm{y}} \\
\eta_{p 2}=\eta_{0}-\frac{B n}{\mathrm{~d} P^{\prime} / \mathrm{d} \xi}, & \text { for } \tau=K \dot{\gamma}-\tau_{\mathrm{y}}
\end{array}
$$

We integrate Equation (9) by applying the following boundary conditions

$$
\begin{aligned}
& u_{x}=U \text { for } y=0 \quad(u=1 \text { for } \eta=0), \\
& u_{x}=U \text { for } y=h(x) \quad(u=1 \text { for } \eta=\tilde{h}) .
\end{aligned}
$$

Furthermore, as the sign of the pressure gradient changes, so does the relation between $\left(\eta_{p 1}, \eta_{p 2}\right)$, and therefore the sign of $\tau_{\mathrm{y}}$ for each flow field in the $y$-direction must be considered. After the appropriate manipulations, the velocity profile is as follows:

- Region with positive pressure gradient $\left(\mathrm{d} P^{\prime} / \mathrm{d} \xi>0\right),\left(\eta_{p 1}>\eta_{p 2}\right)$

$$
u= \begin{cases}1-\left(\frac{\mathrm{d} P^{\prime}}{\mathrm{d} \xi} \eta_{0}+B n\right)(\eta-\tilde{h})+\frac{\mathrm{d} P^{\prime}}{\mathrm{d} \xi} \frac{\left(\eta^{2}-\tilde{h}^{2}\right)}{2}, & \eta_{p 1} \leq \eta \leq \tilde{h} \\ 1-\frac{1}{2} \frac{\mathrm{d} P^{\prime}}{\mathrm{d} \xi} \eta_{0}^{2}+B n \eta_{0}-\frac{B n^{2}}{2\left(\mathrm{~d} P^{\prime} / \mathrm{d} \xi\right)}, & \eta_{p 2} \leq \eta \leq \eta_{p 1} \\ 1+\left(B n-\frac{\mathrm{d} P^{\prime}}{\mathrm{d} \xi} \eta_{0}\right) \eta+\frac{\mathrm{d} P^{\prime}}{\mathrm{d} \xi} \frac{1}{2} \eta^{2}, & 0 \leq \eta \leq \eta_{p 2}\end{cases}
$$

- Region with negative pressure gradient $\left(\mathrm{d} P^{\prime} / \mathrm{d} \xi<0\right),\left(\eta_{p 1}<\eta_{p 2}\right)$

$$
u= \begin{cases}1+\left(-\frac{\mathrm{d} P^{\prime}}{\mathrm{d} \xi} \eta_{0}+B n\right)(\eta-\tilde{h})+\frac{\mathrm{d} P^{\prime}}{\mathrm{d} \xi} \frac{\left(\eta^{2}-\tilde{h}^{2}\right)}{2}, & \eta_{p 2} \leq \eta \leq \tilde{h} \\ 1-\frac{1}{2} \frac{\mathrm{d} P^{\prime}}{\mathrm{d} \xi} \eta_{0}^{2}-B n \eta_{0}-\frac{B n^{2}}{2\left(\mathrm{~d} P^{\prime} / \mathrm{d} \xi\right)}, & \eta_{p 1} \leq \eta \leq \eta_{p 2} \\ 1-\left(\frac{\mathrm{d} P^{\prime}}{\mathrm{d} \xi} \eta_{0}+B n\right) \eta+\frac{\mathrm{d} P^{\prime}}{\mathrm{d} \xi} \frac{1}{2} \eta^{2}, & 0 \leq \eta \leq \eta_{p 1}\end{cases}
$$


At this point, $\eta_{0}$ remains unknown. It can be found by considering the following methodology [7]: for the region with a negative pressure gradient, Equations (13a) for $\eta=\eta_{p 2}$ and (13c) for $\eta=\eta_{p 1}$ should give the same result (plug profile). For both cases, the result of the calculations is:

$$
\eta_{0}=\frac{\tilde{h}}{2},
$$

which suggests that the line where the shear stress equals zero is at the middle of the distance of the web from the roll. This should be expected, since the velocities of the roll and the web are equal.

Integration of the velocity profile gives the dimensionless volumetric flow rate $\lambda$ :

- Region with positive pressure gradient $\left(\mathrm{d} P^{\prime} / \mathrm{d} \xi>0\right),\left(\eta_{p 1}>\eta_{p 2}\right)$

$$
\begin{aligned}
\lambda= & \tilde{h}+\frac{1}{6} \frac{\mathrm{d} P^{\prime}}{\mathrm{d} \xi}\left(\eta_{p 2}^{3}-\eta_{p 1}^{3}\right)+\left(-\frac{\tilde{h}^{2}}{8} \frac{\mathrm{d} P^{\prime}}{\mathrm{d} \xi}-\frac{1}{2} \frac{B n^{2}}{\left(\mathrm{~d} P^{\prime} / \mathrm{d} \xi\right)}+\frac{\tilde{h}}{2} B n\right)\left(\eta_{p 1}-\eta_{p 2}\right) \\
& -\frac{1}{12} \frac{\mathrm{d} P^{\prime}}{\mathrm{d} \xi} \tilde{h}^{3}+\frac{\tilde{h}}{4} \frac{\mathrm{d} P^{\prime}}{\mathrm{d} \xi}\left(\eta_{p 1}^{2}-\eta_{p 2}^{2}\right)+\frac{B n}{2}\left(\eta_{p 1}^{2}+\eta_{p 2}^{2}\right)+\frac{\tilde{h}^{2}}{2} B n-B n h \eta_{p 1}
\end{aligned}
$$

- Region with negative pressure gradient $\left(\mathrm{d} P^{\prime} / \mathrm{d} \xi<0\right),\left(\eta_{p 1}<\eta_{p 2}\right)$

$$
\begin{aligned}
\lambda= & \tilde{h}-\frac{1}{6} \frac{\mathrm{d} P^{\prime}}{\mathrm{d} \xi}\left(\eta_{p 2}^{3}-\eta_{p 1}^{3}\right)+\left(+\frac{\tilde{h}^{2}}{8} \frac{\mathrm{d} P^{\prime}}{\mathrm{d} \xi}+\frac{1}{2} \frac{B n^{2}}{\left(\mathrm{~d} P^{\prime} / \mathrm{d} \xi\right)}+\frac{\tilde{h}}{2} B n\right)\left(\eta_{p 1}-\eta_{p 2}\right) \\
& -\frac{1}{12} \frac{\mathrm{d} P^{\prime}}{\mathrm{d} \xi} \tilde{h}^{3}-\frac{\tilde{h}}{4} \frac{\mathrm{d} P^{\prime}}{\mathrm{d} \xi}\left(\eta_{p 1}^{2}-\eta_{p 2}^{2}\right)-\frac{B n}{2}\left(\eta_{p 1}^{2}+\eta_{p 2}^{2}\right)-\frac{\tilde{h}^{2}}{2} B n+B n h \eta_{p 2}
\end{aligned}
$$

From Equation (15), the pressure gradient $\mathrm{d} P^{\prime} / \mathrm{d} \xi$ can now be found.

Note that for $B n=0$ the above equations and also the velocity profiles - Equations (12a), (12c) and (13a), (13c) - are reduced to the ones given by Middleman [1] for Newtonian fluids.

After numerically calculating the pressure gradients, their integration requires a boundary condition for the pressure $P$. The standard 
condition used in the analysis assumes zero pressure at the separation point [1], i.e.,

$$
P=0 \quad \text { at } \xi=\xi_{1} .
$$

Then the pressure distribution is given by the integral:

$$
P^{\prime}=\int_{-\xi_{f}}^{\xi_{1}}\left\{\frac{\mathrm{d} P^{\prime}}{\mathrm{d} \xi}\right\} \mathrm{d} \xi
$$

or

$$
P^{\prime}=\int_{-\xi_{f}}^{\xi_{1}} I(B n) \mathrm{d} \xi
$$

where $-\xi_{f}$ is the entry point $(=-\infty$ in the case of an infinite reservoir).

Herschel-Bulkley Fluids

The above analysis can be also applied to the viscoplastic Herschel-Bulkley fluids. To do this, it is necessary to derive an equation for the pressure gradient, making use of the constitutive relation (Equation (1)) and the dimensionless generalized Bingham number (Equation (6)) for the expression of the dimensionless yield stress. After the appropriate manipulations, the following velocity profiles are obtained:

- Region with positive pressure gradient $\left(\mathrm{d} P^{\prime} / \mathrm{d} \xi>0\right),\left(\eta_{p 1}>\eta_{p 2}\right)$

$$
u=\left\{\begin{array}{c}
1+\frac{n}{n+1}\left|\frac{\mathrm{d} P^{\prime}}{\mathrm{d} \xi}\right|^{(1 / n)-1} \frac{\mathrm{d} P^{\prime}}{\mathrm{d} \xi}\left\{\left(\eta-\frac{\tilde{h}}{2}-\frac{B n}{\left(\mathrm{~d} P^{\prime} / \mathrm{d} \xi\right)}\right)^{(1 / n)+1}-\left(\frac{\tilde{h}}{2}-\frac{B n}{\left(\mathrm{~d} P^{\prime} / \mathrm{d} \xi\right)}\right)^{(1 / n)+1}\right\}, \\
\eta_{p 1 \leq \eta \leq \tilde{h}} \\
1-\frac{n}{n+1}\left|\frac{\mathrm{d} P^{\prime}}{\mathrm{d} \xi}\right|^{(1 / n)-1} \frac{\mathrm{d} P^{\prime}}{\mathrm{d} \xi}\left(\frac{\tilde{h}}{2}-\frac{B n}{\left(\mathrm{~d} P^{\prime} / \mathrm{d} \xi\right)}\right)^{(1 / n)+1}, \quad \eta_{\mathrm{p} 2 \leq \eta \leq \eta_{\mathrm{p} 1}} \\
1+\frac{n}{n+1}\left|\frac{\mathrm{d} P^{\prime}}{\mathrm{d} \xi}\right|^{(1 / n)-1} \frac{\mathrm{d} P^{\prime}}{\mathrm{d} \xi}\left\{\left(\eta-\frac{\tilde{h}}{2}+\frac{B n}{\left(\mathrm{~d} P^{\prime} / \mathrm{d} \xi\right)}\right)^{(1 / n)+1}-\left(\frac{\tilde{h}}{2}-\frac{B n}{\left(\mathrm{~d} P^{\prime} / \mathrm{d} \xi\right)}\right)^{(1 / n)+1}\right\}, \\
0 \leq \eta \leq \eta_{p 2}
\end{array}\right.
$$


- Region with negative pressure gradient $\left(\mathrm{d} P^{\prime} / \mathrm{d} \xi<0\right),\left(\eta_{p 1}<\eta_{p 2}\right)$

$$
u=\left\{\begin{array}{c}
1+\frac{n}{n+1}\left|\frac{\mathrm{d} P^{\prime}}{\mathrm{d} \xi}\right|^{(1 / n)-1} \frac{\mathrm{d} P^{\prime}}{\mathrm{d} \xi}\left\{\left(\eta-\frac{\tilde{h}}{2}+\frac{B n}{\left(\mathrm{~d} P^{\prime} / \mathrm{d} \xi\right)}\right)^{(1 / n)+1}-\left(\frac{\tilde{h}}{2}+\frac{B n}{\left(d P^{\prime} / d \xi\right)}\right)^{(1 / n)+1}\right\} \\
\eta_{p 2} \leq \eta \leq \tilde{h} \\
1-\frac{n}{n+1}\left|\frac{\mathrm{d} P^{\prime}}{\mathrm{d} \xi}\right|^{(1 / n)-1} \frac{\mathrm{d} P^{\prime}}{\mathrm{d} \xi}\left(\frac{\tilde{h}}{2}+\frac{B n}{\left(\mathrm{~d} P^{\prime} / \mathrm{d} \xi\right)}\right)^{(1 / n)+1}, \quad \eta_{\mathrm{p} 1} \leq \eta \leq \eta_{\mathrm{p} 2} \\
1+\frac{n}{n+1}\left|\frac{\mathrm{d} P^{\prime}}{\mathrm{d} \xi}\right|^{(1 / n)-1} \frac{\mathrm{d} P^{\prime}}{\mathrm{d} \xi}\left\{\left(\eta-\frac{\tilde{h}}{2}-\frac{B n}{\left(\mathrm{~d} P^{\prime} / \mathrm{d} \xi\right)}\right)^{(1 / n)+1}-\left(\frac{\tilde{h}}{2}+\frac{B n}{\left(\mathrm{~d} P^{\prime} / \mathrm{d} \xi\right)}\right)^{(1 / n)+1}\right\} \\
0 \leq \eta \leq \eta_{p 1}
\end{array}\right\}
$$

Equations (18) and (19) are reduced for $n=1$ to the equations of the previous paragraph for Bingham plastics, as they should. Integrating the velocity profiles, we obtain the volumetric flow rate. After the appropriate manipulations these are:

- Region with positive pressure gradient $\left(\mathrm{d} P^{\prime} / \mathrm{d} \xi>0\right),(\tilde{h}-\lambda)>0$

$$
\lambda=\tilde{h}-\frac{2 n}{2 n+1}\left|\frac{\mathrm{d} P^{\prime}}{\mathrm{d} \xi}\right|^{(1 / n)-1}\left(\frac{\mathrm{d} P^{\prime}}{\mathrm{d} \xi}\right)\left(\frac{\tilde{h}}{2}-\frac{B n}{\left(\mathrm{~d} P^{\prime} / \mathrm{d} \xi\right)}\right)^{(1 / n)+1}\left(\frac{\tilde{h}}{2}+\frac{n}{n+1} \frac{B n}{\left(\mathrm{~d} P^{\prime} / \mathrm{d} \xi\right)}\right),
$$

- Region with negative pressure gradient $\left(\mathrm{d} P^{\prime} / \mathrm{d} \xi<0\right),(\tilde{h}-\lambda)<0$

$$
\lambda=\tilde{h}-\frac{2 n}{2 n+1}\left|\frac{\mathrm{d} P^{\prime}}{\mathrm{d} \xi}\right|^{(1 / n)-1}\left(\frac{\mathrm{d} P^{\prime}}{\mathrm{d} \xi}\right)\left(\frac{\tilde{h}}{2}+\frac{B n}{\left(\mathrm{~d} P^{\prime} / \mathrm{d} \xi\right)}\right)^{(1 / n)+1}\left(\frac{\tilde{h}}{2}-\frac{n}{n+1} \frac{B n}{\left(\mathrm{~d} P^{\prime} / d \xi\right)}\right) .
$$

For $n=1$ the above equation reduces to Equation (15), valid for Bingham plastics, while for $B n=0$ it reduces to Equation (7), valid for pseudoplastic fluids obeying the power-law model.

It should be noted that deriving analytically the expressions for the Herschel-Bulkley fluids, one must take into account that $(1 / n)+1$ has to be an even number.

Finally, Equations (16) and (17) are also valid for the HerschelBulkley fluids. 


\section{Yield-line Location}

Once the pressure gradient distribution is obtained, it is easy to calculate the location of the yield lines separating the yielded/unyielded regions. Considering Equations (10a), (10b), and (14), we obtain the following for the dimensionless yield lines:

$$
\begin{aligned}
& \eta_{p 1}=\frac{\tilde{h}}{2}+\frac{B n}{\left|\mathrm{~d} P^{\prime} / \mathrm{d} \xi\right|}, \\
& \eta_{p 2}=\frac{\tilde{h}}{2}-\frac{B n}{\left|\mathrm{~d} P^{\prime} / \mathrm{d} \xi\right|} .
\end{aligned}
$$

\section{Sheet Thickness}

The entering sheet thickness $H_{f}$ is given by:

$$
\frac{H_{f}}{H_{0}}=1+\frac{1}{2} \xi_{f}^{2},
$$

and in the case of a flooded (infinite) reservoir, it is infinity (or a very big number).

The resulting exit coating thickness is given by:

$$
\frac{H}{H_{0}}=\frac{\lambda}{2} \text {. }
$$

The analysis is still not complete at this point, since a relation between the dimensionless flow rate $\lambda$, and the separation point $\xi_{1}$, should be found, as in the case of pseudoplastic fluids [1]. The same boundary conditions are assumed, i.e., the velocity vanishes at the separation point (the first stagnation point), which explains the formation of a meniscus. Moreover, as Figure 1 suggests, the film splits evenly, so that the separation point is at $\left(\xi_{1}, \tilde{h} / 2\right)$. This assumption can be accepted due to the fact that the roll and the sheet move with the same velocity. In any other case, the surface moving faster would take up a bigger part of the material.

In the case of viscoplastic fluids, the corresponding equation found analytically connects the separation point $\xi_{1}$ with the dimensionless flow rate $\lambda$ and the rheological parameters, as expected. It should be mentioned though, that since it also includes the unknown value of the 
pressure gradient $\mathrm{d} P^{\prime} / \mathrm{d} \xi$ at the separation point, it cannot be used in the same manner as Equation (8) and so the problem requires a numerical solution.

In particular, and regarding the more general case of viscoplastic Herschel-Bulkley fluids, the expression connecting the separation point $\xi_{1}$, with the dimensionless coating thickness $\lambda$, and the power-law index $n$, is (by combining Equations (18b) and (20a) and setting the velocity equal to zero):

$$
\xi_{1}=\left(2\left(\frac{2 n+1}{n} \lambda-1+\frac{2 B n}{\mathrm{~d} P^{\prime} / \mathrm{d} \xi}\right)\right)^{1 / 2} .
$$

For $B n=0$, Equation (24) reduces to Equation (8) valid for pseudoplastic fluids obeying the power-law model.

\section{Operating Variables}

Once the pressure gradient and pressure distributions are found as functions of $n$ or $B n$, then all other quantities of interest are readily available. The operating variables used in engineering calculations are computed in the following manner [1]:

(i) the maximum pressure, $\mathbf{P}(n, B n)$, defined by:

$$
\mathbf{P}(n, B n)=\int_{-[2(\lambda-1)]^{0.5}}^{\xi_{1}} I(n, B n) \mathrm{d} \xi,
$$

where $-[2(\lambda-1)]^{0.5}$ corresponds to the point where the pressure reaches a maximum.

(ii) the roll-separating force per unit width $W, \mathrm{~F} / \mathrm{W}(n, B n)$, defined by:

$$
\frac{F}{W}=\int_{-x_{f}}^{x_{1}} P(x) \mathrm{d} x=K\left(\frac{U}{H_{0}}\right)^{n} R F(n, B n)
$$

with

$$
\mathbf{F}(n, B n)=\int_{-\xi_{f}}^{\xi_{1}}\left[\int_{\xi}^{\xi_{1}} I(n, B n) \mathrm{d} \xi\right] \mathrm{d} \xi
$$

(iii) the power input, $\dot{\boldsymbol{W}}(n, B n)$, defined by:

$$
\dot{\boldsymbol{W}}(n, B n)=\left.W U \int_{-x_{f}}^{x_{1}} \tau_{x y}\right|_{y=h(x)} \mathrm{d} x=\frac{W U K}{2}\left(\frac{U}{H_{0}}\right)^{n} \sqrt{R H_{0}} \mathbf{E}(n, B n),
$$


with

$$
\mathbf{E}(n, B n)=\int_{-\xi_{f}}^{\xi_{1}} I(n, B n)\left(1+\frac{1}{2} \xi^{2}\right) \mathrm{d} \xi
$$

(iv) the adiabatic temperature rise, $(\Delta T)_{\text {ave }}$, defined by:

$$
(\Delta T)_{\mathrm{ave}}=\frac{\dot{\boldsymbol{W}}(n, B n)}{Q \rho c_{p}},
$$

where $\rho$ is the melt density and $c_{p}$ is the melt heat capacity.

\section{METHOD OF SOLUTION}

The governing equation (20) for the pressure gradient is nonlinear and has no analytical solution for the general case of viscoplastic materials, even for the Bingham plastics. Thus, it requires a numerical solution based on some appropriate algorithm.

The input data for the present work are the rheological parameters of the fluid, i.e., the power-law index $n$, and the Bingham number $B n$, along with the entry point $-\xi_{f}$. Since this work is concerned with the case of a flooded upstream region (infinite reservoir), a big number was selected, which after a series of numerical tests was set equal to -100 . Another numerical aspect concerns the number of intervals, in which the flow field is subdivided. This number has to be sufficiently high so that the numerical results are accurate. We have tested $10^{5}$ and $10^{6}$ intervals, because of the highly nonlinear equation, especially for cases of extreme non-Newtonian behavior, such as small values of the powerlaw index $n$ and high values of the Bingham number $B n$. The numerical solutions with $10^{6}$ intervals were virtually the same with results with $10^{5}$ intervals. Therefore, there is sufficient numerical accuracy in the present results. The tolerance in our numerical algorithm for convergence is set to $10^{-9}$.

\section{Pseudoplastic Fluids}

We have used the Modified Regula-Falsi (MRF) numerical method [8] to solve the governing equation for the pressure gradient. The numerical integration of these values to obtain the pressure distribution is done via Simpson's rule. In order to find the $\lambda$-value for which the pressure 
is zero at the separation point, we use again the MRF method. The separation point $\xi_{1}$ is expressed as a function of $\lambda$ and $n$, using Equation (8).

\section{Viscoplastic Fluids}

In this case, both the separation point and the dimensionless flow rate have to be calculated. The problem is solved as in the previous case, with the added feature of an external loop, based on the MRF method, which keeps changing the separation point, until there exists a pair of values $\left(\lambda, \xi_{1}\right)$ for which both the fluid velocity and the pressure are zero at the separation point.

It should be noted that this algorithm was first tested for the pseudoplastic fluids, for which the relation between $\left(\lambda, \xi_{1}\right)$ is explicitly given by Equation (8). More specifically, the MRF method was used for the calculation of the separation point, disregarding Equation (8). A comparison for the results $\left(\lambda, \xi_{1}\right)$ between the two methods (Equation (8) and numerical solution) showed that the maximum difference was $0.043 \%$ for $\lambda$ and $0.025 \%$ for $\xi_{1}$. Thus, confidence was established in the numerical algorithm to also use it for viscoplastic fluids.

The yield lines are calculated via Equations $(21 a, b)$ using the pressure gradient values. Finally, the exit coating thickness $H / H_{0}$ is calculated from Equation (23) for all cases.

\section{RESULTS AND DISCUSSION}

\section{Pseudoplastic Fluids}

First the calculations are pursued for pseudoplastic power-law fluids, both shear-thinning $(0 \leq n \leq 1)$ and shear-thickening $(1 \leq n \leq 2)$. The numerical results for the volumetric flow rate $\lambda$, the exit coating thickness $H / H_{0}$, and the separation point $\xi_{1}$, are presented in Table 1 . Numerical results for the maximum pressure and the minimum pressure gradient are given in Table 2.

The results for the dimensionless pressure gradient distribution are shown in Figure 4, while the results for the dimensionless pressure distribution are shown in Figure 5. The smaller the powerlaw index $n$ the bigger the dimensionless maximum pressure, and the steeper the pressure gradient curves. Also, the smaller the power-law index $n$ the bigger the flow domain, and the bigger the exit coating thickness. 
Table 1. Dimensionless volumetric flow rate $\lambda$, exit coating thickness $H / H_{0}$, and separation point $\xi_{1}$, for roll-over-web coating pseudoplastic power-law fluids from an infinite reservoir.

\begin{tabular}{lccc}
\hline $\boldsymbol{n}$ & $\lambda$ & $\boldsymbol{H} / \boldsymbol{H}_{\mathbf{0}}$ & $\boldsymbol{\xi}_{\mathbf{1}}$ \\
\hline 0.1 & 1.6395 & 0.8197 & 6.1113 \\
0.2 & 1.5208 & 0.7604 & 4.3921 \\
0.3 & 1.4526 & 0.7263 & 3.6735 \\
0.4 & 1.4083 & 0.7041 & 3.2672 \\
0.5 & 1.3771 & 0.6885 & 3.0028 \\
0.6 & 1.3540 & 0.6770 & 2.8159 \\
0.7 & 1.3363 & 0.6681 & 2.6764 \\
0.8 & 1.3223 & 0.6611 & 2.5680 \\
0.9 & 1.3109 & 0.6554 & 2.4813 \\
1.0 & 1.3015 & 0.6507 & 2.4102 \\
1.1 & 1.2936 & 0.6468 & 2.3508 \\
1.2 & 1.2869 & 0.6435 & 2.3005 \\
1.3 & 1.2811 & 0.6406 & 2.2573 \\
1.4 & 1.2761 & 0.6381 & 2.2198 \\
1.5 & 1.2717 & 0.6359 & 2.1869 \\
1.6 & 1.2678 & 0.6339 & 2.1578 \\
1.7 & 1.2644 & 0.6322 & 2.1319 \\
1.8 & 1.2613 & 0.6306 & 2.1087 \\
1.9 & 1.2585 & 0.6293 & 2.0878 \\
2.0 & 1.2560 & 0.6280 & 2.0688 \\
\hline
\end{tabular}

This last observation is verified in Figure 6, which shows the dimensionless volumetric flow rate $\lambda$, and the exit coating thickness $H / H_{0}$, as a function of the power-law index $n$. The well-known Newtonian value (for $n=1$ ) of $\lambda=1.3015$ [3] is a starting point, after which it is noted that shear-thinning increases the values, reaching for $n=0.1, \lambda=1.6395$. On the other hand, shear-thickening decreases the values, reaching for $n=2, \lambda=1.2560$. Thus, the maximum coating thickness $H / H_{0}$, can be as high as 0.8197 for extremely shear-thinning fluids $(n=0.1), 0.6507$ for Newtonian fluids $(n=1)$, and 0.6280 for shear-thickening fluids with $n=2$.

In Figure 7 the separation point $\xi_{1}$, and the exit coating thickness $H / H_{0}$, are depicted, as obtained from a full parametric range of the power-law index $n$. We observe that the smaller the power-law index, the bigger the values of the separation point, and thus the bigger the flow domain.

Finally, Figure 8 shows the engineering quantities $\mathbf{P}, \mathbf{F}$, and $\mathbf{E}$. A decrease of the power-law index $n$ increases these dimensionless quantities. The Newtonian values $(n=1)$ are $\mathbf{F}=2.5959, \mathbf{P}=1.9208$, and $\mathbf{E}=10.4141$. 
Table 2. Dimensionless maximum pressure $\boldsymbol{P}_{\text {max }}^{\prime}$ and minimum pressure gradient $d P^{\prime} /\left.d \xi\right|_{\xi=0}$, for roll-over-web coating pseudoplastic power-law fluids from an infinite reservoir.

\begin{tabular}{lcc}
\hline $\boldsymbol{n}$ & $\boldsymbol{P}_{\text {max }}^{\prime}$ & $\mathrm{d} \boldsymbol{P}^{\prime} /\left.\mathrm{d} \xi\right|_{\xi=0}$ \\
\hline 0.1 & 2.5816 & -2.6281 \\
0.2 & 2.6074 & -2.9757 \\
0.3 & 2.5530 & -3.2075 \\
0.4 & 2.4610 & -3.3631 \\
0.5 & 2.3748 & -3.4737 \\
0.6 & 2.2752 & -3.5437 \\
0.7 & 2.1831 & -3.5894 \\
0.8 & 2.0915 & -3.6126 \\
0.9 & 2.0041 & -3.6218 \\
1.0 & 1.9220 & -3.6173 \\
1.1 & 1.8430 & -3.6045 \\
1.2 & 1.7690 & -3.5836 \\
1.3 & 1.6991 & -3.5566 \\
1.4 & 1.6331 & -3.5249 \\
1.5 & 1.5718 & -3.4887 \\
1.6 & 1.5117 & -3.4507 \\
1.7 & 1.4558 & -3.4096 \\
1.8 & 1.4028 & -3.3666 \\
1.9 & 1.3525 & -3.3221 \\
2.0 & 1.3059 & -3.2756 \\
\hline
\end{tabular}

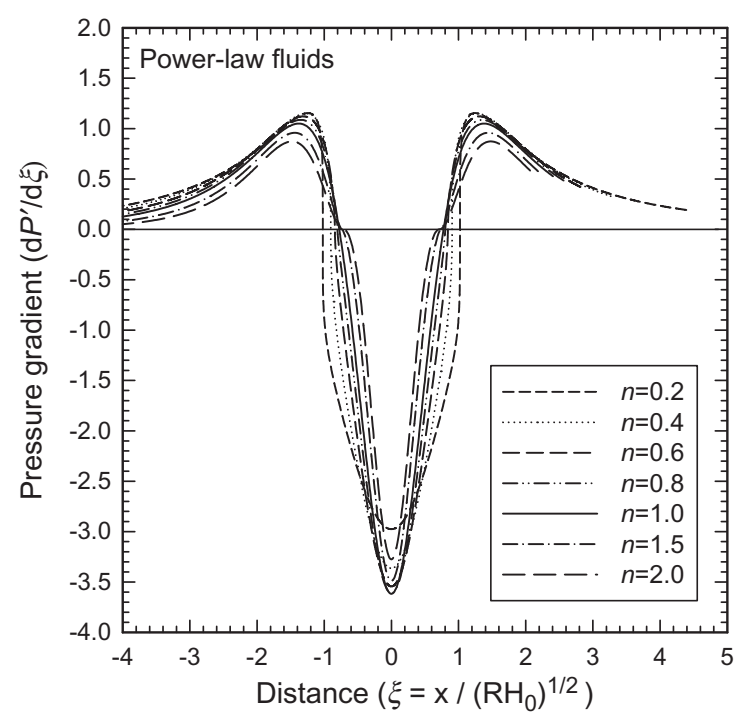

Figure 4. Axial distribution of dimensionless pressure gradient for different values of the power-law index $n$, for pseudoplastic fluids. 


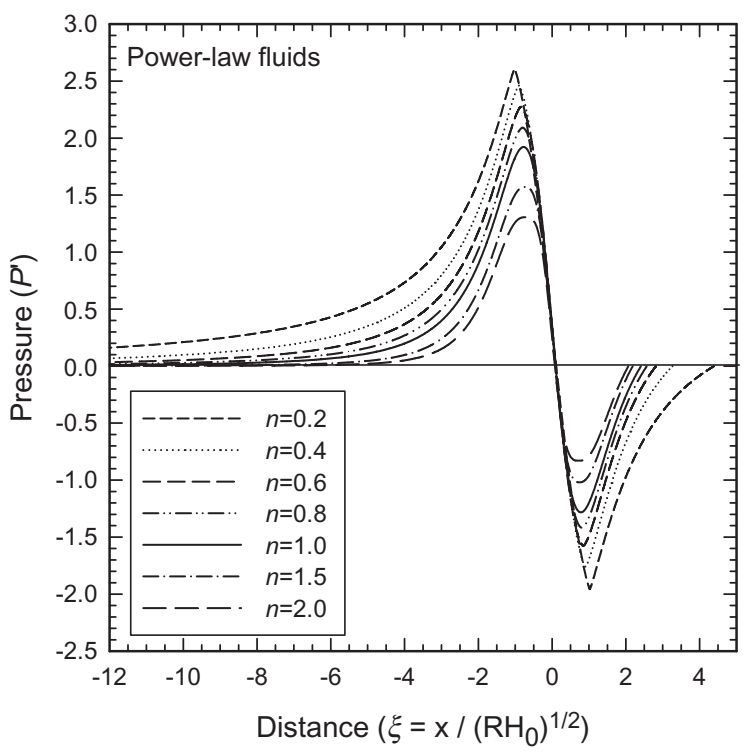

Figure 5. Axial distribution of dimensionless pressure for different values of the powerlaw index $n$, for pseudoplastic fluids.

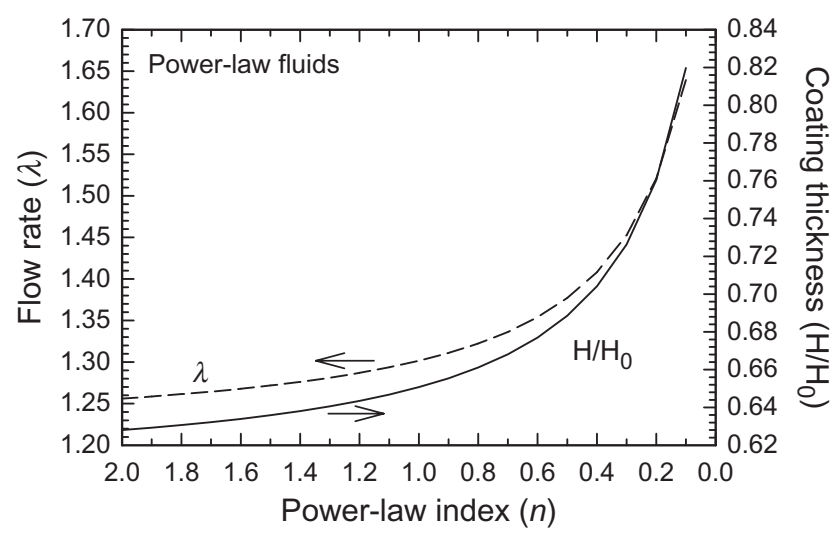

Figure 6. Dimensionless flow rate $\lambda$, and exit coating thickness $H / H_{0}$, as a function of the power-law index $n$, for pseudoplastic fluids.

\section{Viscoplastic Fluids}

Calculations were performed next for viscoplastic fluids, and in particular for Bingham plastics, with $0 \leq B n \leq 15$. Numerical results for the dimensionless volumetric flow rate $\lambda$, final coating thickness $H / H_{0}$, 


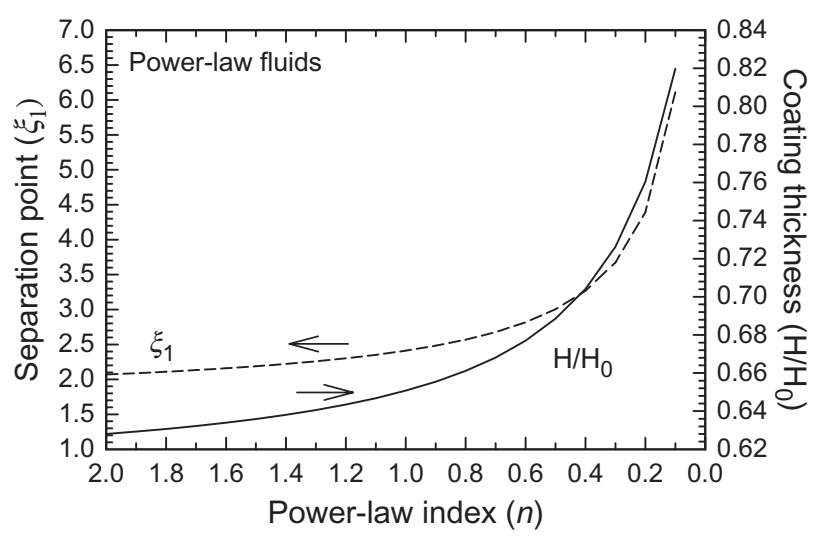

Figure 7. Dimensionless separation point $\xi_{1}$, and exit coating thickness $H / H_{0}$, as a function of the power-law index $n$, for pseudoplastic fluids.

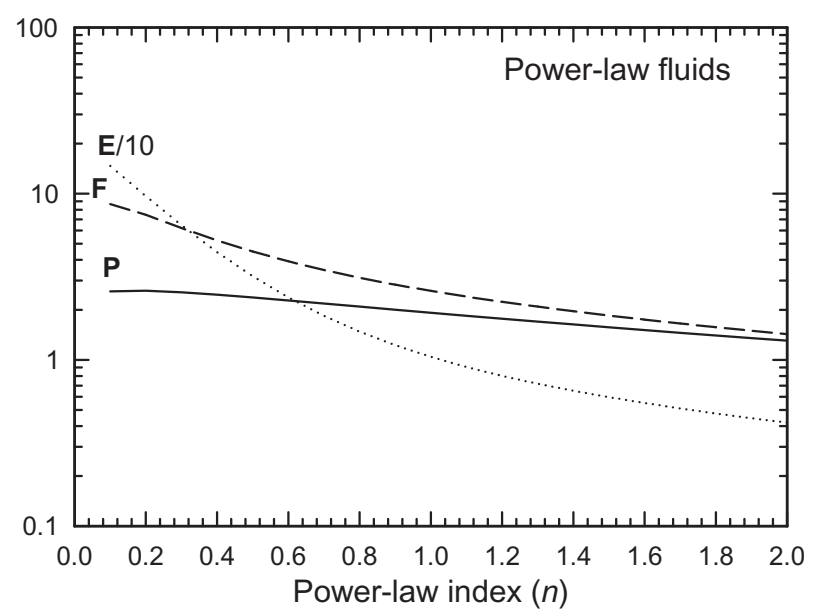

Figure 8. Operating variables (maximum pressure $\mathbf{P}$, force factor $\mathbf{F}$, and power factor $\mathbf{E}$ ) as a function of the power-law index $n$, for pseudoplastic fluids.

and separation point $\xi_{1}$, are presented in Table 3 , while numerical results for the maximum pressure and the minimum pressure gradient are presented in Table 4.

Figure 9 shows the dimensionless pressure gradient distribution for different values of the Bingham number $B n$, while Figure 10 shows the corresponding dimensionless pressure distribution. As was the case for pseudoplastic shear-thinning fluids, increasing $B n$ leads to higher values for the maximum pressure, while the curves for the pressure gradient 
Table 3. Dimensionless volumetric flow rate $\lambda$, exit coating thickness $H / H_{0}$, and separation point $\xi_{1}$, for roll-over-web coating viscoplastic Bingham fluids from an infinite reservoir.

\begin{tabular}{lccc}
\hline $\mathbf{B n}$ & $\boldsymbol{\lambda}$ & $\boldsymbol{H} / \boldsymbol{H}_{\mathbf{0}}$ & $\xi_{\mathbf{1}}$ \\
\hline $\mathbf{0}$ & $\mathbf{1 . 3 0 1 5}$ & $\mathbf{0 . 6 5 0 7}$ & $\mathbf{2 . 4 1 0 2}$ \\
0.001 & 1.3017 & 0.6508 & 2.4120 \\
0.01 & 1.3032 & 0.6516 & 2.4281 \\
0.05 & 1.3098 & 0.6549 & 2.4990 \\
0.1 & 1.3175 & 0.6588 & 2.5858 \\
0.5 & 1.3666 & 0.6833 & 3.2252 \\
1 & 1.4095 & 0.7048 & 3.9239 \\
2 & 1.4673 & 0.7337 & 5.1167 \\
3 & 1.5068 & 0.7534 & 6.1393 \\
4 & 1.5364 & 0.7682 & 7.0504 \\
5 & 1.5602 & 0.7801 & 7.8825 \\
6 & 1.5798 & 0.7899 & 8.6529 \\
7 & 1.5966 & 0.7983 & 9.3749 \\
8 & 1.6109 & 0.8055 & 10.0556 \\
9 & 1.6236 & 0.8118 & 10.7023 \\
10 & 1.6349 & 0.8175 & 11.3205 \\
11 & 1.6449 & 0.8224 & 11.9118 \\
12 & 1.6540 & 0.8270 & 12.4811 \\
13 & 1.6623 & 0.8312 & 13.0311 \\
14 & 1.6703 & 0.8352 & 13.5662 \\
15 & 1.6773 & 0.8387 & 14.0824 \\
\hline
\end{tabular}

are getting steeper near the minimum gap $H_{0}$. Thus, as the material becomes more viscoplastic, the deformations in the flow domain become smaller, the material behaves more solid-like and requires higher pressures and a larger domain to flow and deform.

Figure 11 shows the values for the dimensionless volumetric flow rate $\lambda$, and the exit coating thickness $H / H_{0}$, as a function of $B n$. For $B n=0$ the well-known Newtonian value $\lambda=1.3015$ [3] is the starting point for the calculations of viscoplastic fluids. Further increasing $B n$ leads to higher increases of these values. For $B n=10$, the coating thickness $H / H_{0}$, is 0.8175 .

Figure 12 shows the separation point $\xi_{1}$, and the coating thickness $H / H_{0}$, for $0 \leq B n \leq 15$. Increasing $B n$, and hence the viscoplastic character of the material, leads again to higher values of the separation point, and hence the flow domain.

Figure 13 shows results for the operating variables, $\mathbf{P}, \mathbf{F}$, and $\mathbf{E}$. For $B n=0$, the Newtonian values are obtained which were mentioned above, while increasing $B n$ leads again to increasing these values, in a dimensionless form. 
Table 4. Dimensionless maximum pressure $P_{\text {max }}^{\prime}$ and minimum pressure gradient $d P^{\prime} /\left.d \xi\right|_{\xi=0}$, for roll-over-web coating viscoplastic Bingham fluids from an infinite reservoir.

\begin{tabular}{lrr}
\hline $\mathbf{B n}$ & \multicolumn{1}{c}{$\boldsymbol{P}_{\text {max }}^{\prime}$} & $\mathbf{d} \mathbf{P}^{\prime} /\left.\mathbf{d} \xi\right|_{\xi=\mathbf{0}}$ \\
\hline $\mathbf{0}$ & $\mathbf{1 . 9 2 2 0}$ & $-\mathbf{3 . 6 1 7 3}$ \\
0.001 & 1.9249 & -3.6231 \\
0.01 & 1.9607 & -3.6686 \\
0.05 & 2.1173 & -3.8676 \\
0.1 & 2.3092 & -4.1101 \\
0.5 & 3.7422 & -5.8849 \\
1 & 5.3861 & -7.8493 \\
2 & 8.4357 & -11.3585 \\
3 & 11.3218 & -14.5733 \\
4 & 14.1018 & -17.6115 \\
5 & 16.8330 & -20.5375 \\
6 & 19.4974 & -23.3781 \\
7 & 22.1351 & -26.1503 \\
8 & 24.7420 & -28.8744 \\
9 & 27.3298 & -31.5531 \\
10 & 29.8877 & -34.1975 \\
11 & 32.4316 & -36.8075 \\
12 & 34.9645 & -39.3960 \\
13 & 37.4763 & -41.9551 \\
14 & 39.9998 & -44.5009 \\
15 & 42.4852 & -47.0197 \\
\hline
\end{tabular}

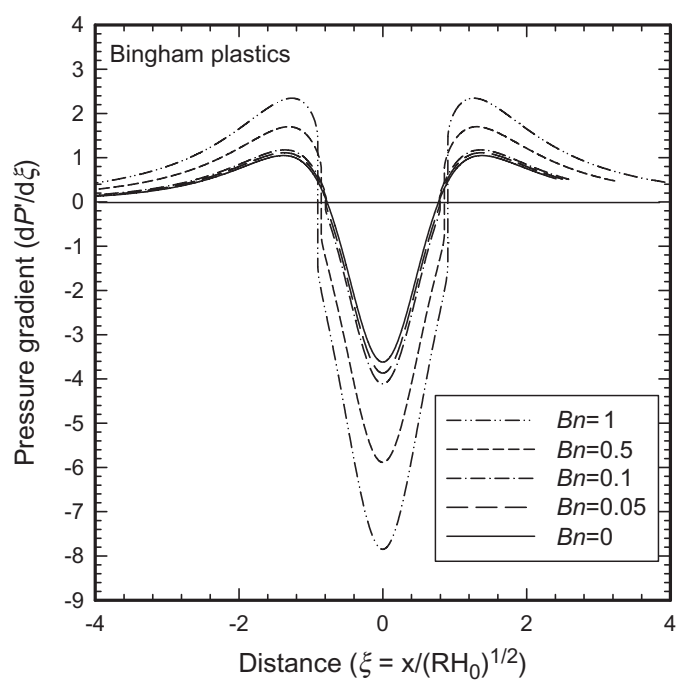

Figure 9. Axial distribution of dimensionless pressure gradient for different values of the Bingham number $B n$, for viscoplastic fluids. 


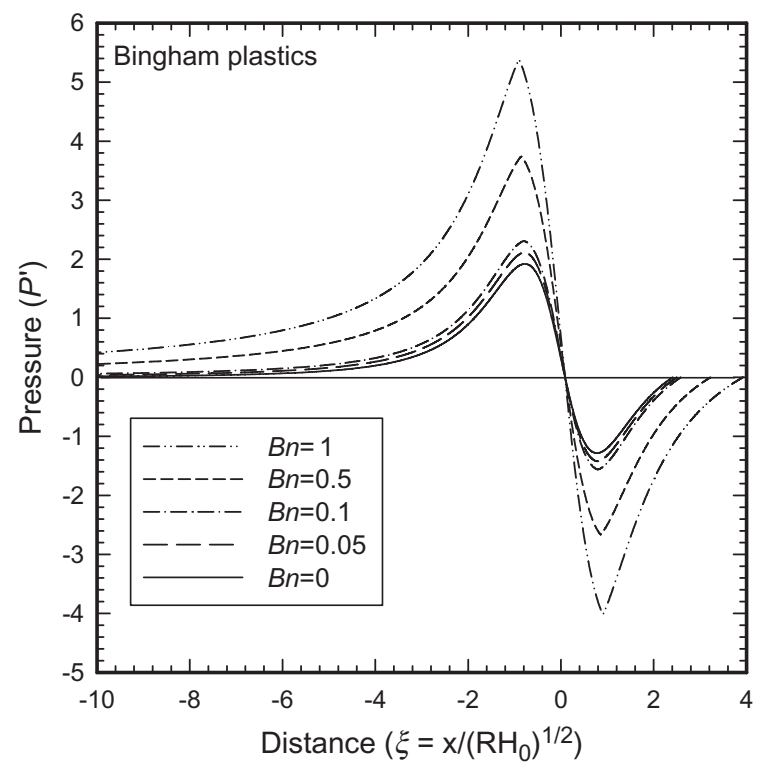

Figure 10. Axial distribution of dimensionless pressure for different values of the Bingham number $B n$, for viscoplastic fluids.

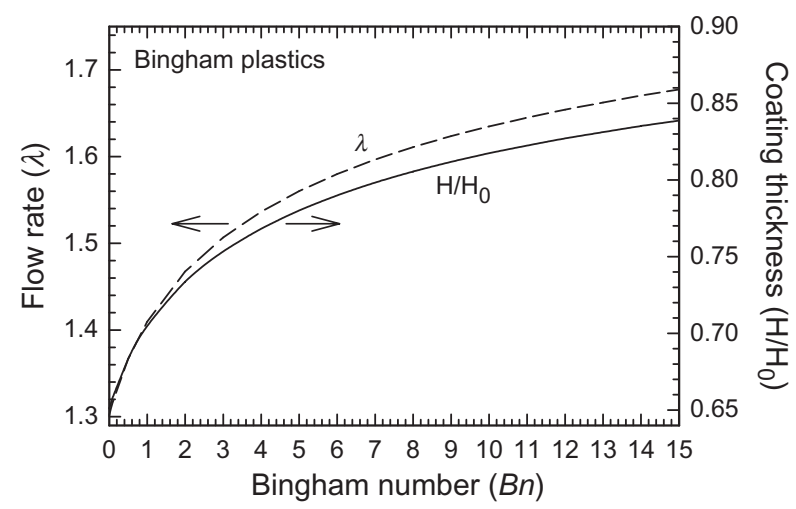

Figure 11. Dimensionless flow rate $\lambda$, and exit coating thickness $H / H_{0}$, as a function of the Bingham number $B n$, for viscoplastic fluids.

Figure 14 shows the yielded and unyielded regions for various values of $\mathrm{Bn}$. The dashed line corresponds to the 'symmetry' line of the present problem, or in other words the locus of the points where the shear stress is zero. It should be noted that the two yield lines are symmetric with regard to this line, as evidenced from Equations (21a) and (21b). 


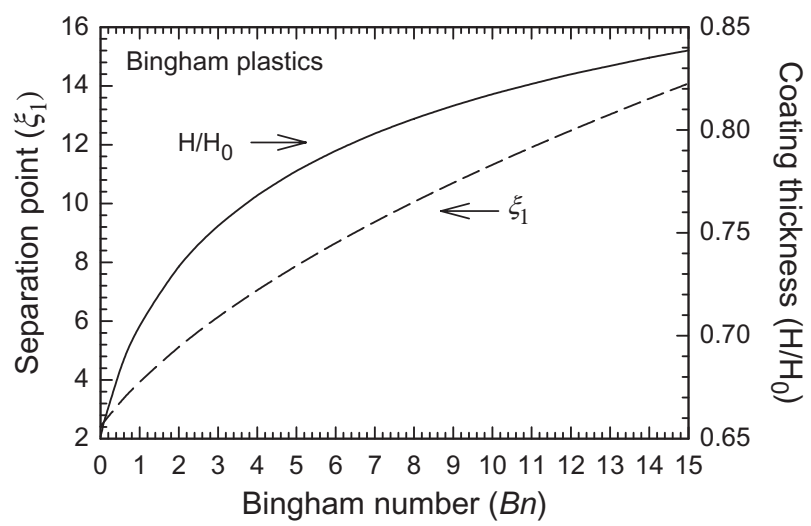

Figure 12. Dimensionless separation point $\xi_{1}$, and exit coating thickness $H / H_{0}$, as a function of the Bingham number $B n$, for viscoplastic fluids.

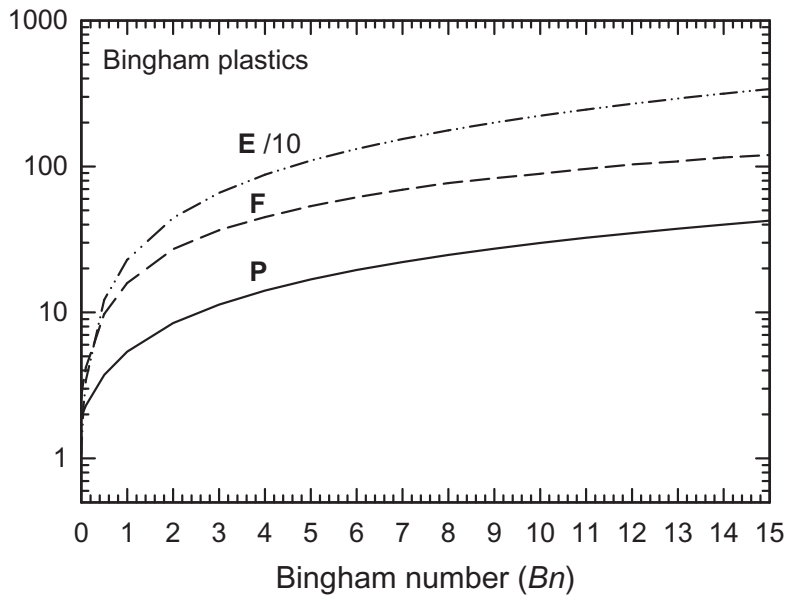

Figure 13. Operating variables (maximum pressure $\mathbf{P}$, force factor $\mathbf{F}$, and power factor $\mathbf{E}$ ) as a function of the Bingham number $B n$, for viscoplastic fluids.

Increasing the $B n$ number changes the shape and extent of the yielded/unyielded regions. In particular, increasing $B n$ (a dimensionless yield stress) leads to a progressive development of unyielded regions. The points (on the flow axis) having a plug velocity profile, i.e., $\left\{\eta_{p 1}=\tilde{h}, \eta_{p 2}=0\right\}$, are those for which the pressure shows extrema: $\xi_{\alpha 1}=[2(\lambda-1)]^{0.5}, \xi_{\alpha 2}=-[2(\lambda-1)]^{0.5}$. Thus, as the increase in $B n$ leads to higher $\lambda$ values, these points get farther away from the minimum gap $H_{0}$, which produces a gradual change in the shape of the unyielded regions. 

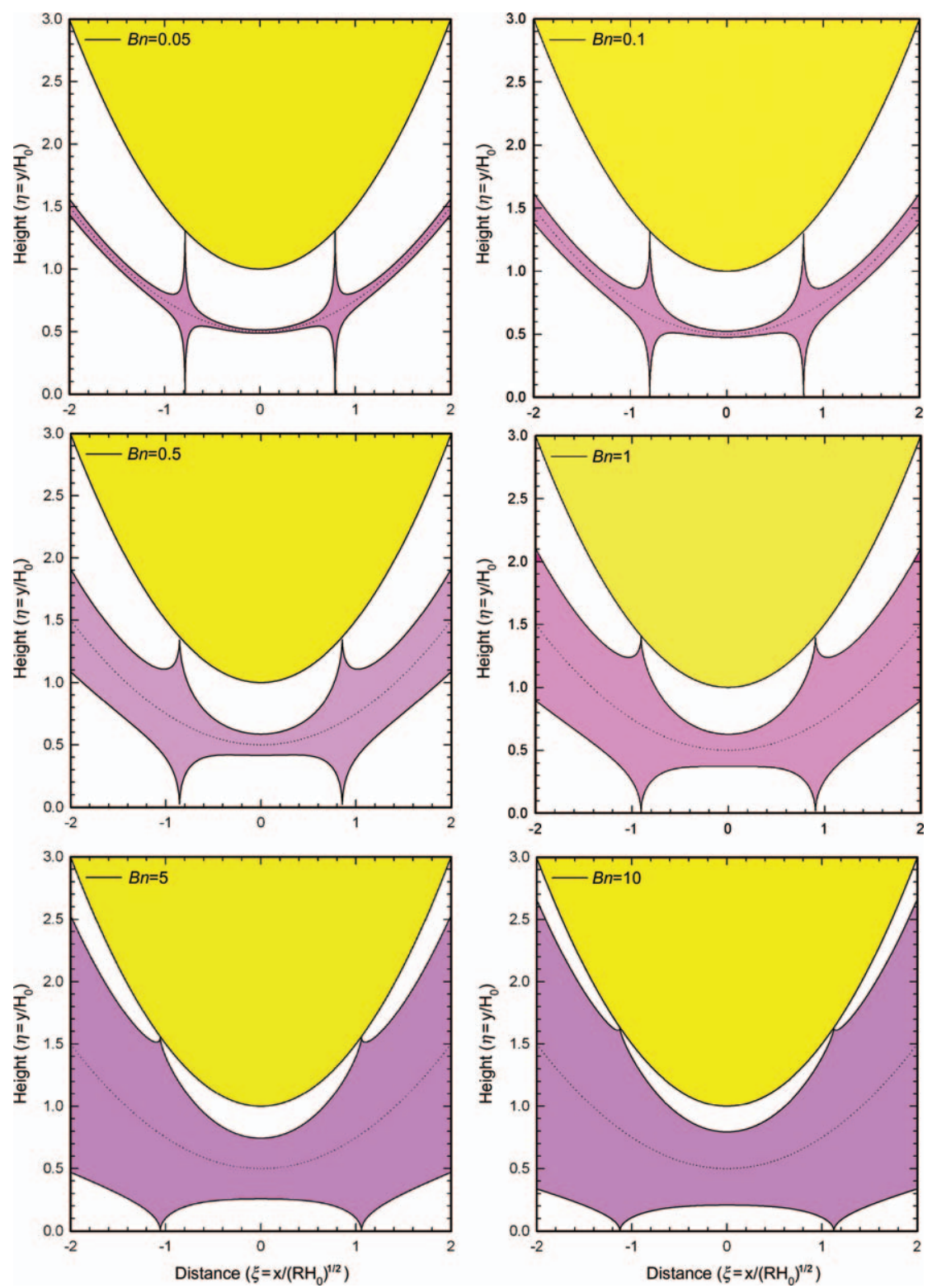

Figure 14. Progressive growth of the unyielded zones as the dimensionless Bingham number $B n$, increases in roll-over-web coating of viscoplastic materials obeying the Bingham plastic model. The shaded regions are unyielded. 
The parametric study for Bingham plastics would have been complete, if a $B n$ number were found for which the velocity profile were plug for the whole flow field, something which is not observed for $B n=15$. However, it is noteworthy that a comparison of the viscoplastic results for $B n \approx 10$ for the coating thickness and the dimensionless flow rate with those for the pseudoplastic shear-thinning fluids with $n=0.1$ revealed that these are about the same. On the other hand, the separation point is much larger for viscoplastic fluids. After a series of numerical tests, it became clear that a further increase in the $B n$ number led to very high values of the separation point (see Table 3 ), but it did not lead to a fully plug velocity profile and did not increase the values for the coating thickness and the volumetric flow rate accordingly. This can be explained by the fact that for large values of the separation point, the flow domain cannot be considered as that between two flat plates, and the assumptions of LAT, $u_{y} \ll u_{x}$ and $\mathrm{d} x \ll \mathrm{d} y$, are not valid. We have, therefore, come across the limitations of LAT for extracting useful numerical results.

The effect of pseudoplasticity within viscoplasticity is examined in Figure 15, where the pressure distribution is depicted for several Herschel-Bulkley fluids at $B n=1$. Changing the value of the power-law index $n$, has no dramatic effect over the maximum pressure value,

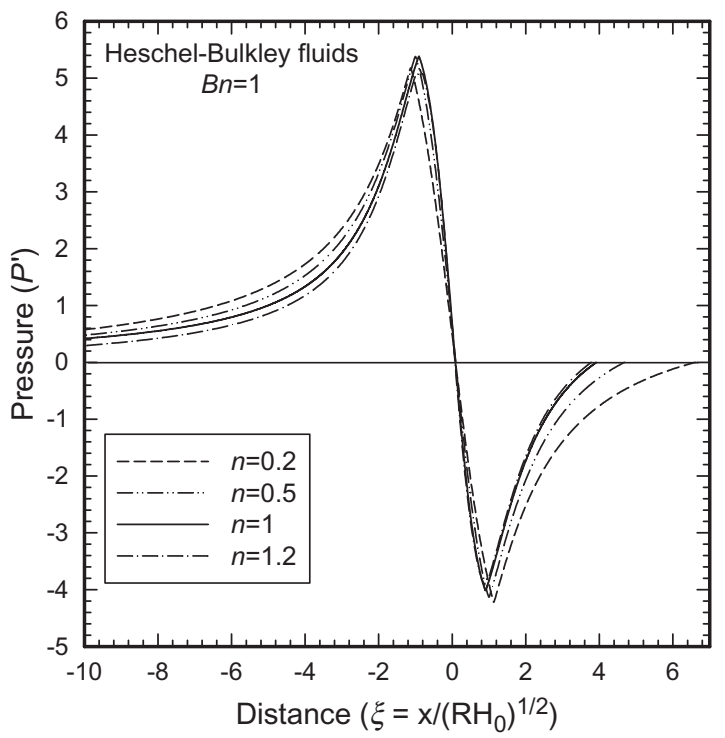

Figure 15. Axial distribution of dimensionless pressure for different values of the powerlaw index $n$, for Herschel-Bulkley fluids with $B n=1$. 
and it does not change the shape of the pressure curve. The decrease of the power-law index does however lead to a bigger domain, and bigger values for the dimensionless flow rate $\lambda$. For $n=0.2$, the acquired values for $\lambda$ and $\xi_{1}$ are 1.642 and 6.618 respectively, that is, $16.5 \%$ and $68.6 \%$ bigger than the ones presented in Table 3 for a Bingham plastic with $B n=1$. Once again, the sensitivity of $\xi_{1}$ is more pronounced. These results confirm that the meniscus location is one of the most sensitive features in modeling this type of flow.

\section{Critique on the Lubrication Approximation}

The key assumption for obtaining the above results has been the lubrication approximation theory (LAT), which assumes locally fully developed flow and reduces the conservation equations by using only the axial velocity component. This assumption is known to give good results for the pressure distribution in calendering of power-law fluids [9] and hence for all integrated quantities resulting from that. It is therefore reasonable to assume that it also does well for viscoplastic fluids, such as the ones used in the present work.

However, a closer look at the physics of the viscoplastic problem and in particular the interesting yielded/unyielded regions found here, reveals that these cannot be so and that Figure 14 is contentious. The shaded areas cannot be rigid plugs, since the speeds at entry and exit are different, and there is no chance that a constant speed occurs along the line where the shear stress equals zero. However, the domain length, the pressure distribution, and the ensuing operating variables are not expected to change much (within a few percent) from the values found by LAT, as was the case in calendering [10].

It is therefore obvious that the introduction of LAT in lubrication flows with viscoplastic fluids is not valid for such quantities as the yielded/ unyielded regions, since it leads to a 'paradox', as pointed out by Lipscomb and Denn [11]. A 2-D analysis is therefore essential for obtaining the correct regions, and such an analysis is currently under way by the authors. However, it is not expected to change drastically the other results shown here, especially the pressure distribution and integrated quantities, since roll-over-web coating is primarily a lubrication flow.

\section{CONCLUSIONS}

In the present work, the Lubrication Approximation Theory (LAT) was used along with the power-law, Bingham, and Herschel-Bulkley 
rheological models, in order to obtain numerical results for the process of roll coating over a moving flat web fed from an infinite reservoir. For the pseudoplastic fluids, the results by Middleman [1,6] were verified and extended, while for viscoplastic fluids, a new methodology was presented and gave new results for Bingham plastics and Herschel-Bulkley fluids.

Reducing the power-law index $n$, or increasing the Bingham number $B n$, leads to higher coating thickness, separation point (hence domain length) and volumetric flow rate. Furthermore, engineering quantities of the process, such as the maximum pressure, the separating force and the required power to the roll, all increase with a reduction in $n$ or an increase in $B n$. Finally, the determination of yielded/unyielded regions showed a progressive growth of unyielded regions by increasing the $B n$ number. It was argued that this last finding is a direct result of the approximation within LAT, and that these unyielded regions should disappear in a full 2-D analysis of the process. Such an analysis remains to be done for roll-over-web coating of viscoplastic fluids.

The present results are offered as a quick reference for engineers working on roll-over-web coating of such materials to determine the resulting coating thickness of the coatings produced and the corresponding values for important engineering quantities.

\section{ACKNOWLEDGMENTS}

Financial support from NTUA in the form of a research scholarship for basic research for S. Sofou is gratefully acknowledged.

\section{REFERENCES}

1. Middleman, S. (1977). Fundamentals of Polymer Processing, McGraw-Hill, New York.

2. Bird, R.B., Dai, G.C. and Yarusso, B.J. (1983). The Rheology and Flow of Viscoplastic Materials, Rev. Chem. Eng., 1(1): 1-70.

3. Coyle, D.J. (1984). The Fluid Mechanics of Roll Coating: Steady Flows, Stability, and Rheology, PhD Thesis, University of Minnesota, Minneapolis, MN.

4. Coyle, D.J., Macosko, C.W. and Scriven, L.E. (1987). Film-Splitting Flows of Shear-Thinning Liquids in Forward Roll Coating, AIChE J., 33(3): 741-746.

5. Coyle, D.J., Macosko, C.W. and Scriven, L.E. (1990). Reverse Roll Coating of Non-Newtonian Liquids, J. Rheol., 34(5): 615-636. 
6. Greener, J. and Middleman, S. (1975). A Theory of Roll Coating of Viscous and Viscoelastic Fluids, Polym. Eng. Sci., 15(1): 1-10.

7. Loest, H., Lipp, R. and Mitsoulis, E. (1994). Numerical Flow Simulation of Viscoplastic Slurries and Design Criteria for a Tape Casting Unit, J. Amer. Ceram. Soc., 77(1): 254-262.

8. Gerald, C.F. and Wheatley, P.O. (1985). Applied Numerical Analysis, Addison-Wesley, Reading, Massachusetts.

9. Mitsoulis, E., Vlachopoulos, J. and Mirza, F.A. (1985). Calendering Analysis without the Lubrication Approximation, Polym. Eng. Sci., 25(1): 6-18.

10. Sofou, S. and Mitsoulis, E. (2004). Calendering of Pseudoplastic and Viscoplastic Sheets of Finite Thickness, J. Plast. Film \& Sheeting, 20(3): 185-222.

11. Lipscomb, G.G. and Denn, M.M. (1984). Flow of Bingham Fluids in Complex Geometries, J. Non-Newtonian Fluid Mech., 14(1): 337-346.

\section{BIOGRAPHIES}

\section{Evan Mitsoulis}

Evan Mitsoulis received his Diploma in Chemical Engineering from the National Technical University of Athens (NTUA), Greece, before obtaining his MScE from the University of New Brunswick (Canada) and then his $\mathrm{PhD}$ in ChE from McMaster University (Canada). From 1984-2000 he was a Professor in the Department of Chemical Engineering at the University of Ottawa, Canada, and since 1998 he has been with the School of Mining Engineering and Metallurgy at NTUA, where he is the Director of CAMP-R\&D (Computer-Aided Materials Processing - Rheology \& Design) Laboratory. He is the author of over 100 refereed papers and over 185 presentations in Conferences on polymer processing, rheology, and design.

\section{Souzanna Sofou}

Souzanna Sofou received her Diploma in Mining Engineering \& Metallurgy from the National Technical University of Athens (NTUA), Greece, before obtaining her MEng at the same University. She is now a $\mathrm{PhD}$ candidate in the School of Mining Engineering and Metallurgy at NTUA working on "Calendering of Viscoplastic Materials". 